Bundesgesundheitsbl 2013 $\cdot 56: 755-764$

DOI 10.1007/s00103-013-1691-8

Online publiziert: 27. Mai 2013

(c) Springer-Verlag Berlin Heidelberg 2013

\section{Additional material online}

An English full-text version of this article is available at SpringerLink under supplementary material: dx.doi.org/10.1007/s00103-013-1691-8
R. Schlack ${ }^{1} \cdot$ J. Rüdel ${ }^{2} \cdot$ A. Karger ${ }^{3} \cdot$ H. Hölling ${ }^{1}$

${ }^{1}$ Abteilung für Epidemiologie und Gesundheitsmonitoring, Robert Koch-Institut, Berlin

2 Fakultät für Sozialwissenschaft, Ruhr-Universität Bochum

${ }^{3}$ Klinisches Institut für Psychosomatische Medizin und Psychotherapie, Universität Düsseldorf

\title{
Körperliche und psychische Gewalterfahrungen in der deutschen Erwachsenenbevölkerung
}

\section{Ergebnisse der Studie zur Gesundheit Erwachsener in Deutschland (DEGS1)}

Gewalt ist - je nach Breite der Definition - ein ubiquitäres soziales Phänomen. Neben staatlicher und kollektiver Gewalt, beispielsweise Diktatur, Staatsterrorismus, Kriegs- oder Bürgerkriegszustände [1], steht mit Blick auf die Alltagssituation die individuelle Gewalt im öffentlichen oder privaten Raum im Zentrum des Interesses. Selg et al. [2] verstehen unter Gewalt schwere Formen von Aggression, bei denen zusätzlich zu einer Schädigungsabsicht ein psychisches oder physisches Machtgefälle zugrunde liegt. Häufig wird zwischen direkter (körperlicher) und indirekter (verbaler/psychischer und sozial-relationaler, d. h. auf die Beschädigung sozialer Beziehungen gerichteter) Gewalt unterschieden. Hinsichtlich individueller Gewalt lässt sich zudem nach den Sozialräumen, innerhalb derer sich Gewalthandlungen vollziehen, unterscheiden: häusliche Gewalt, d. h. Gewalt durch oder gegen den Partner oder andere Familienangehörige, Gewalt am Arbeitsplatz oder Gewaltereignisse zwischen sich bekannten oder zuvor unbekannten Personen. Gewalterfahrungen in der Kindheit und Jugend sind mit einem höheren Risiko verbunden, im späteren Lebensalter erneut Opfer von Gewalt zu werden, aber auch mit einem erhöhten Risiko, selbst Gewalt auszuüben $[3,4]$. Gewalterfahrungen sind mit erheblichen psychosozialen und gesundheitlichen Konsequenzen wie körperlichen und seelischen Verletzungen, Rückzug und Isolation, Depression, Angststörungen, sozialen Beeinträchtigungen bei den Opfern, aber auch Delinquenz, strafrechtlicher Verfolgung, Gefängnisaufenthalten, Persönlichkeitsstörungen, Depression, Substanzkonsum sowie schlechteren Berufs- und Bildungschancen bei Opfern und Tätern verbunden $[5,6,7,8]$, wodurch eine erhebliche Public-Health-Relevanz begründet ist. Es ist davon auszugehen, dass Gewalt als Risikofaktor für eine Vielzahl von körperlichen und psychischen, zum Teil lang anhaltenden Gesundheitsstörungen in den Gesundheitswissenschaften immer noch unterschätzt wird [9]. Statistiken (z. B. die polizeiliche Kriminalstatistik) und Studien - vor allem zur häuslichen Gewalt weisen oft mehrheitlich Männer als Gewalttäter aus [10, 11]. Dagegen ist kritisch eingewendet worden, dass die Ergebnisse solcher Studien durch nichtrepräsentative Stichproben (z. B. Inanspruchnahmestichproben mit Teilnehmer/innen aus Unfallambulanzen oder Frauenhäusern) sowie durch unscharfe Begriffsdefinitionen oder einseitige Fokussierung auf körperliche Gewalt oder auf Opfererfahrungen von Frauen systematisch verzerrt sein könnten $[12,13,14]$. Es erschien daher als gute epidemiologische Praxis für einen bundesweit repräsentativen Gesundheitssurvey, mindestens sowohl körperliche als auch psychische Gewalt als Tä- ter- und Opfererfahrung bei beiden Geschlechtern in verschiedenen Sozialräumen zu erheben. Ziel dieses Beitrags ist es, die geschlechtsspezifischen Häufigkeiten und kontextspezifischen Verteilungen körperlicher und psychischer Gewaltopfererfahrung sowie Gewaltausübung in der deutschen Erwachsenenbevölkerung $\mathrm{zu}$ berichten und den Zusammenhang mit Belastungs- und Unrechtserleben zu untersuchen.

\section{Methoden}

\section{Stichprobe und Methodik}

Die „Studie zur Gesundheit Erwachsener in Deutschland" (DEGS) ist Bestandteil des Gesundheitsmonitorings des Robert Koch-Instituts (RKI). Konzept und Design von DEGS sind an anderer Stelle ausführlich beschrieben $[15,16,17,18,19]$. Die erste Erhebungswelle (DEGS1) wurde von 2008 bis 2011 durchgeführt und umfasste Befragungen, Untersuchungen und Tests [20, 21]. Zielpopulation war die in Deutschland lebende Bevölkerung im Alter von 18 bis 79 Jahren. DEGS1 hat ein Mischdesign, das gleichzeitig querund längsschnittliche Analysen ermöglicht. Hierbei wurde eine Einwohnermeldeamtsstichprobe durch ehemalige Teilnehmerinnen und Teilnehmer des Bundes-Gesundheitssurveys 1998 (BGS98) ergänzt. Insgesamt nahmen 8152 Personen 
teil, darunter 4193 Ersteingeladene (Response $42 \%$ ) und 3959 ehemalige Teilnehmerinnen und Teilnehmer des BGS98 (Response 62\%). 7238 Personen besuchten eines der 180 Untersuchungszentren, 914 wurden ausschließlich befragt. Die Nettostichprobe [19] ermöglicht für den Altersbereich von 18 bis 79 Jahren repräsentative Querschnittanalysen und Trendaussagen im Vergleich mit dem BGS98 $(\mathrm{n}=7988$, davon 7116 in Untersuchungszentren).

Aktive und passive Erfahrungen körperlicher und psychischer Gewalt wurden im Altersbereich von 18 bis 64 Jahren bei insgesamt 5939 Teilnehmerinnen und Teilnehmern, davon 3149 Frauen und 2790 Männer (ungewichtete Angaben) erhoben. Da Gewalterfahrungen im BGS98 nicht thematisiert waren, präsentiert dieser Beitrag ausschließlich querschnittliche Daten. Die Analysen wurden mit einem Gewichtungsfaktor durchgeführt, der Abweichungen der Stichprobe von der Bevölkerungsstruktur (Stand 31.12.2010) hinsichtlich Alter, Geschlecht, Region und Staatsangehörigkeit sowie Gemeindetyp und Bildung korrigiert [19]. Für den Untersuchungsteil wurde ein gesonderter Gewichtungsfaktor erstellt. Um sowohl die Gewichtung als auch die Korrelation der Teilnehmenden innerhalb einer Gemeinde zu berücksichtigen, wurden die Konfidenzintervalle und p-Werte mit den SPSS-20-Verfahren für komplexe Stichproben bestimmt. Nicht überlappende 95\%-Konfidenzintervalle werden als signifikante Unterschiede auf dem $\mathrm{p}<0,05$-Niveau gewertet. Der Sozialstatus wurde anhand eines Indexes bestimmt, in den Angaben zu schulischer und beruflicher Ausbildung, beruflicher Stellung sowie Haushaltsnettoeinkommen (bedarfsgewichtet) eingehen und der eine Einteilung in niedrige, mittlere und hohe Statusgruppe ermöglicht [22].

Die Erhebung von Gewalterfahrungen erfordert eine besondere Berücksichtigung ethischer Aspekte. Neben der verfahrensförmigen Absicherung durch Einbeziehung einer Ethikkommission sind insbesondere die Sicherheit und die Anonymität der befragten Personen, eine intensive Schulung und Betreuung des Studienpersonals sowie vorbereitete Maßnahmen für durch die Be- fragung potenziell ausgelöste Retraumatisierungen der Teilnehmenden zu nennen [23]. In DEGS1 war die Sicherheit der Teilnehmenden dadurch gewährleistet, dass die Befragung in einem außerhäuslichen Untersuchungszentrum im Rahmen der Gesundheitsuntersuchungen durchgeführt wurde. Die Fragen zur Gewalt waren in den schriftlichen Selbstbeurteilungsfragebogen integriert. Die Fragebögen waren nicht namentlich gekennzeichnet. Das Studienpersonal wurde initial inhaltlich geschult und kontinuierlich nachgeschult und wurde von der Feldleitung, einer internen sowie einer unabhängigen externen Qualitätssicherung engmaschig begleitet und betreut [24]. Anfängliche Überlegungen, auch Erfahrungen sexueller Gewalt in die Erhebung einzubeziehen, wurden aufgrund der eingeschränkten Möglichkeiten zur Krisenintervention im Untersuchungssetting nicht realisiert. Für den Fall psychischer Belastungen durch die Befragung waren für jeden Untersuchungsort im Voraus aktuelle Kontakttelefone und Notfalladressen recherchiert worden, die den jeweiligen Untersuchungsteams zur Verfügung standen.

\section{Instrumente}

Ein konzises, aussagekräftiges und Survey-geeignetes Screening-Instrument zu Gewalterfahrungen wurde in der Vorbereitungsphase trotz intensiver Literaturrecherche nicht gefunden. Daher wurde im RKI mit beratender Unterstützung der Universität Bielefeld ein Screener zu körperlicher und psychischer Gewalt entwickelt und im Pretest des DEGS1 erfolgreich getestet. Parallel zur DEGS-Erhebung wurde der Gewalt-Screener an einer Stichprobe von 830 Patienten, die sich in der Ambulanz der Klinik für Psychosomatische Medizin und Psychotherapie der Universität Düsseldorf zu einem Erstgespräch vorstellten, klinisch validiert. Die Validierungsstudie befindet sich derzeit in der Auswertung.

Alle Fragen zur aktuellen Betroffenheit von Gewalt beziehen sich auf die letzten 12 Monate. Fragebogendramaturgisch wurden zunächst körperliche Gewalterfahrungen und anschließend psychische Gewalterfahrungen erfragt. Um psychische Schließungseffekte zu vermeiden, wurden jeweils zunächst Opfer- und dann Tätererfahrungen erfragt. Der Fragenblock begann mit einer Eingangssequenz, die in allgemeiner Form auf Gewalterfahrungen verwies, ohne jedoch das Wort "Gewalt" explizit zu nennen. Diese lautete: „Manchmal werden Menschen angegriffen, geraten in körperliche Auseinandersetzungen oder machen Erfahrungen, die sie als verletzend oder belastend empfinden. In den folgenden Fragen geht es um diese speziellen Erfahrungen in Ihrem Alltag."

\section{Körperliche Gewalt}

Anschließend folgte eine Einstiegsfrage, in der eine in vielen Gewaltstudien übliche Definition von körperlicher Gewalt präsentiert wurde [25]. Diese Frage lautete für die Viktimisierung durch körperliche Gewalt: „Haben Sie in den letzten 12 Monaten erlebt, dass jemand Sie körperlich angegriffen hat (zum Beispiel geschlagen, geohrfeigt, an den Haaren gezogen, getreten, mit einer Waffe oder mit einem Gegenstand bedroht)?", bzw. für die Ausübung körperlicher Gewalt: „Haben Sie selbst in den letzten 12 Monaten jemanden körperlich angegriffen (zum Beispiel geschlagen, geohrfeigt, an den Haaren gezogen, getreten, mit einer Waffe oder mit einem Gegenstand bedroht)?“

\section{Psychische Gewalt}

Für die Viktimisierung durch psychische Gewalt lautete die Einstiegsfrage: „Sind Sie in den letzten 12 Monaten durch irgendeinen Menschen abgewertet worden (z. B. bezüglich Ihres Aussehens, Ihrer Art, sich zu kleiden, Ihrer Denk-, Handlungs- oder Arbeitsweise oder möglicher Behinderungen)? Oder wurden Sie beleidigt, beschimpft, bedroht, schikaniert oder unter Druck gesetzt?", und für Tätererfahrungen psychischer Gewalt: „Haben Sie selbst in den letzten 12 Monaten irgendeinen Menschen abgewertet (z. B. bezüglich Ihres Aussehens, Ihrer Art, sich zu kleiden, Ihrer Denk-, Handlungsoder Arbeitsweise oder möglicher Behinderungen)? Oder haben Sie eine Person beleidigt, beschimpft, bedroht, schikaniert oder unter Druck gesetzt?" Die Antwortkategorien lauteten jeweils „Ja“ und „Nein“. 
Bundesgesundheitsbl 2013 ·56:755-764 DOI 10.1007/s00103-013-1691-8

(c) Springer-Verlag Berlin Heidelberg 2013

\section{R. Schlack · J. Rüdel · A. Karger $\cdot$ H. Hölling}

\section{Körperliche und psychische Gewalterfahrungen in der deutschen Erwachsenenbevölke- rung. Ergebnisse der Studie zur Gesundheit Erwachsener in Deutschland (DEGS1)}

\section{Zusammenfassung}

Gewalterfahrungen sind von erheblicher Public-Health-Relevanz. Das Ziel des in die Studie zur Gesundheit Erwachsener in Deutschland (DEGS1) implementierten Gewaltscreenings war es, erstmalig in einem bundesweit repräsentativen Gesundheitssurvey Daten zu körperlicher und psychischer Gewalt in unterschiedlichen Sozialräumen (Partnerschaft, Familie, Arbeitsplatz, öffentlicher Raum) aus der Opfer- und Täterperspektive sowohl bei Männern als auch bei Frauen zu erheben. Die Studienpopulation bestand aus 5939 Teilnehmenden im Alter von 18 bis 64 Jahren. Körperliche Gewaltopfererfahrungen in den letzten 12 Monaten berichtete insgesamt etwa jeder 20. Teilnehmende, Männer signifikant häufiger als Frauen. Hinsichtlich der Häufigkeit von Tätererfahrungen körperlicher Ge- walt (insgesamt 3,7\%) gab es keine signifikanten Geschlechtsunterschiede. Psychische Gewaltopfererfahrungen berichtete jeder fünfte, Tätererfahrungen psychischer Gewalt insgesamt jeder zehnte Teilnehmende. Frauen waren tendenziell häufiger Opfer, jedoch waren sie signifikant häufiger Täterinnen von körperlicher und psychischer Gewalt im häuslichen Bereich (Partnerschaft, Familie). Männer gaben dagegen häufiger an, am Arbeitsplatz sowie im öffentlichen Raum sowohl Täter als auch Opfer gewesen zu sein. Junge Erwachsene von 18 bis 29 Jahren sowie Personen mit niedrigem Sozialstatus waren durchgängig häufiger von Gewalt betroffen, wobei es Ausnahmen hinsichtlich psychischer Gewalterfahrungen gab. Mehr als drei Viertel der Opfer von körperlicher Gewalt ga- ben an, dadurch in ihrem Befinden stark oder sehr stark beeinträchtigt zu sein, bei psychischer Gewalt waren dies ca. 60\%. Das Belastungserleben infolge körperlicher und psychischer Gewaltopfererfahrungen war bei Männern insgesamt deutlich höher, insbesondere bei häuslicher Gewalt (Partnerschaft, Familie). Frauen gaben insgesamt ein höheres Unrechtserleben nach Gewaltausübung an als Männer; hinsichtlich der Ausübung von Partnergewalt gab es hier jedoch keine Geschlechtsunterschiede.

\section{Schlüsselwörter}

Körperliche und psychische Gewalt . Partnergewalt - Gewalt am Arbeitsplatz . Gewalt im öffentlichen Raum . Gesundheitssurvey

\section{Physical and psychological violence perpetration and violent victimisation in the German adult population. Results of the German Health Interview and Examination Survey for Adults (DEGS1)}

\section{Abstract}

Violence is of considerable relevance to Public Health. It was the aim of the violence screening implemented as part of the German Health Interview and Examination Survey for Adults (DEGS1) to assess data on physical and psychological violence in various social environments (partnership, family, workplace, public space). For the first time as part of a nationally representative health survey, the data were collected from the perspective of victim and perpetrator both among women and men. The study population was comprised of 5,939 participants aged between 18 and 64 years. Approximately every 20th participant reported being the victim of physical violence in the preceding 12 months, men significantly more frequently than women. With regard to the frequency of being the perpetrator of physical violence (overall prevalence $3.7 \%$ ) there were no significant differences between the sexes. Psychological victimisation was reported by every fifth participant and overall perpetrating psychological violence was reported by every tenth. Women tended to be more frequently the victims but they were also significantly more frequently the perpetrators of both physical and psychological violence in the domestic area (partnership, family). In contrast, men more frequently reported being both the perpetrator and the victim of violence in the workplace and in public spaces. Young adults between 18 and 29 years as well as persons of low socioeconomic status were consistently more frequently affected by violence although there were exceptions with regard to psychological victimisation. More than three-quarters of the victims of physical violence reported being greatly or extremely affected in their well-being by the violence and in the case of psychological violence the rate was about approximately $60 \%$. Impairments in well-being following physical and psychological violence were considerably higher in men, especially in the case of domestic violence (partnership, family). Overall, women reported a greater sense of wrongdoing following violence perpetration than men; as to the perpetration of violence towards a partner, however, there was no difference between the sexes in this regard. An English full-text version of this article is available at SpringerLink as supplemental.

\section{Keywords}

Physical and psychological violence . Intimate partner violence . Workplace violence - Violence in the public space . Health survey

\section{Sozialraum/Konfliktpartner}

Wurde die jeweilige Einstiegsfrage zur körperlichen bzw. psychischen Gewalterfahrung bejaht, sollten weitere Fragen nach möglichen Konfliktpartnern beantwortet werden. Die Antwortmöglichkeiten waren: „Partnerin/Partner“, „Person aus der Familie/sonstige Verwandtschaft", „Kolleg(inn)en/Vorgesetzte am Arbeits- platz“, „sonstige bekannte Person“, „sonstige (zuvor) unbekannte Person“. Mehrfachangaben waren möglich.

\section{Belastungserleben durch Viktimisierung}

Zusätzlich wurde sowohl bei körperlicher als auch bei psychischer Viktimisierung mit folgendem Item nach dem Belastungs- erleben in Zusammenhang mit der widerfahrenen Gewalt gefragt: „Wie stark fühlen oder fühlten Sie sich dadurch in Ihrem Befinden beeinträchtigt?" Die Antwortkategorien lauteten „Gar nicht“, „Kaum“, „Etwas“, „Stark“ oder „Sehr stark“. Für die vorliegenden Analysen wurden die Kategorien „Stark“ und „Sehr stark“ zusammengefasst. 
Tab. 1 12-Monats-Prävalenz körperlicher Gewalterfahrungen bei 18- bis 64-jährigen Frauen und Männern nach Altersgruppen und Sozialstatus in Deutschland (DEGS1)

\begin{tabular}{|lllllll}
\hline & \multicolumn{5}{c}{ Gewalt widerfahren } & \multicolumn{3}{l}{ Gewalt ausgeübt } \\
\cline { 2 - 6 } & Frauen \% (95\%-KI) & Männer \% (95\%-KI) & Gesamt \% (95\%-KI) & Frauen \% (95\%-KI) & Männer \% (95\%-KI) & Gesamt \% (95\%-KI) \\
\hline Gesamt & $3,3(2,6-4,2)$ & $6,2(5,0-7,6)$ & $4,8(4,1-5,6)$ & $3,4(2,7-4,4)$ & $3,9(3,0-4,9)$ & $3,7(3,1-4,3)$ \\
\hline Altersgruppen & & & & & \\
\hline $\begin{array}{l}18 \text { bis } \\
\text { 29 Jahre }\end{array}$ & $7,7(5,6-10,6)$ & $17,0(13,6-21,1)$ & $12,5(10,4-14,9)$ & $6,1(4,1-9,1)$ & $10,6(7,8-14,2)$ & $8,4(6,6-10,6)$ \\
\hline $\begin{array}{l}\text { 30 bis } \\
\text { 44 Jahre }\end{array}$ & $1,6(0,9-2,8)$ & $2,2(1,3-3,8)$ & $1,9(1,3-2,8)$ & $5,2(3,7-7,3)$ & $2,4(1,4-4,0)$ & $3,8(2,9-5,0)$ \\
\hline $\begin{array}{l}\text { 45 bis } \\
\text { 64 Jahre }\end{array}$ & $1,9(1,3-2,8)$ & $1,6(0,9-2,8)$ & $1,9(1,3-2,8)$ & $0,8(0,4-1,4)$ & $1,4(0,8-2,4)$ & $1,1(0,7-1,6)$ \\
\hline Sozialstatus & & & & & \\
\hline Niedrig & $6,3(3,9-10,0)$ & $9,5(6,5-13,7)$ & $7,9(6,0-10,4)$ & $2,9(1,4-5,6)$ & $5,7(3,6-8,8)$ & $4,3(3,0-6,1)$ \\
\hline Mittel & $3,0(2,1-4,2)$ & $6,1(4,5-8,1)$ & $4,5(3,6-5,6)$ & $4,0(2,9-5,4)$ & $3,9(2,7-5,5)$ & $3,9(3,1-4,9)$ \\
\hline Hoch & $1,8(1,0-3,1)$ & $3,4(2,1-5,5)$ & $2,7(1,8-3,8)$ & $2,3(1,4-3,8)$ & $2,5(1,4-4,3)$ & $2,4(1,7-3,4)$ \\
\hline KI Konfidenzintervall. & & & & &
\end{tabular}

Tab. 2 12-Monats-Prävalenz psychischer Gewalterfahrungen bei 18- bis 64-jährigen Frauen und Männern in Deutschland (DEGS1)

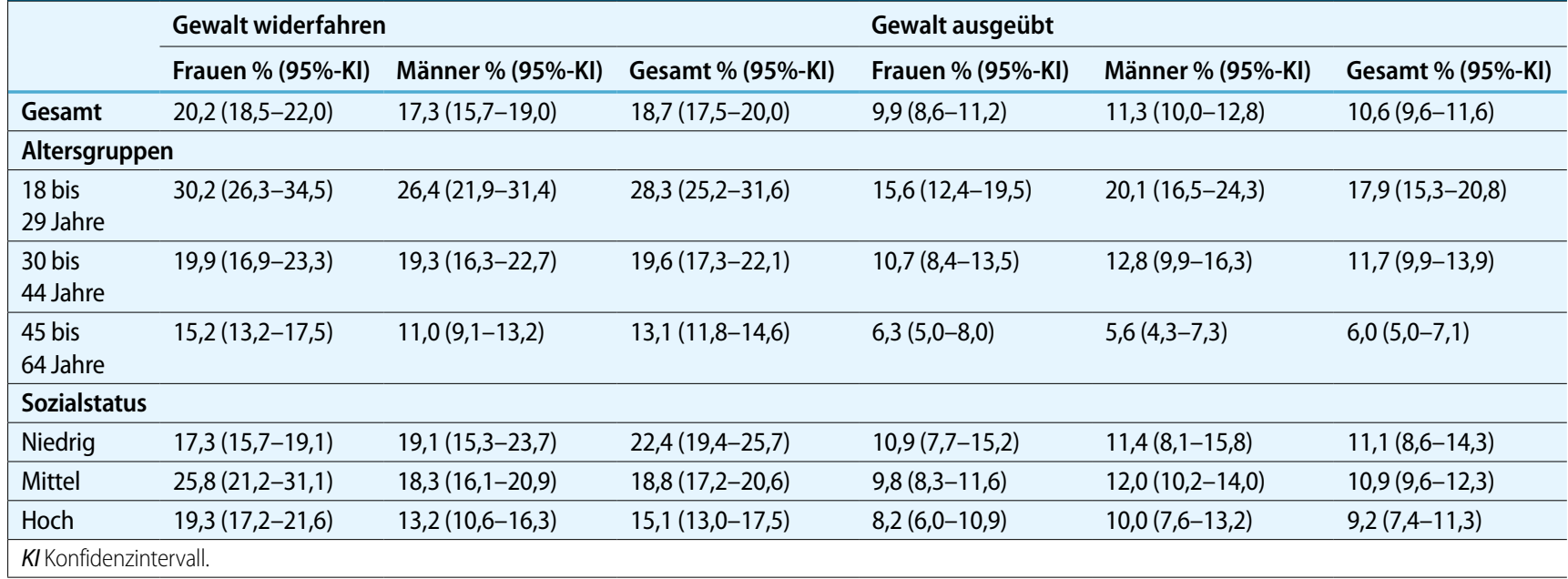

\section{Unrechtserleben nach Gewaltausübung}

Sowohl hinsichtlich körperlicher als psychischer Tätererfahrungen wurde gefragt: „Haben Sie deswegen schon einmal ein schlechtes Gewissen gehabt oder sich schuldig gefühlt?"“ Die Antwortmöglichkeiten lauteten jeweils „Ja“ und „Nein“.

\section{Ergebnisse}

\section{Körperliche Gewalt nach \\ Geschlecht, Alter und \\ Sozialstatus}

Die Prävalenz körperlicher Gewaltopfererfahrungen in den letzten 12 Monaten in der deutschen Erwachsenenbevölkerung von 18 bis 64 Jahren liegt insgesamt bei 4,8\% (• Tab. 1). Frauen gaben mit 3,3\% signifikant seltener an, in den letzten 12 Monaten Opfer von körperlicher Gewalt gewesen zu sein als Männer $(6,2 \%)$. Selbst körperliche Gewalt ausgeübt zu haben, gaben insgesamt 3,4\% der Frauen und 3,9\% der Männer an. Der Unterschied ist nicht signifikant. Im Altersgang nehmen sowohl Opfer- als auch Täterprävalenzen für körperliche Gewalt deutlich ab. Vor allem das junge Erwachsenenalter (18 bis 29 Jahre) erweist sich als stark gewaltbelastet. Eine stärkere Belastung von Personen mit niedrigem Sozialstatus durch körperliche Gewalt (Opfer- und Tätererfahrung) zeigt sich darüber hinaus als klarer Trend. Dabei sind die Unterschiede sowohl bei Frauen als auch bei Männern mit niedrigem gegenüber Frauen und Männern mit hohem Sozialstatus signifikant.
Psychische Gewalt nach

Geschlecht, Alter und

Sozialstatus

Die Prävalenzen für eine Viktimisierung durch psychische Gewalt liegen deutlich höher als diejenigen für körperliche $\mathrm{Ge}$ walt, für beide Geschlechter bei ca. $20 \%$ (• Tab. 2). In den letzten 12 Monaten Ausübende psychischer Gewalt gewesen zu sein, gaben Männer und Frauen etwa gleich häufig mit ca. 9\% an.

Auch hinsichtlich psychischer Gewalt (sowohl Täter- als auch Opfererfahrungen) ist die Gruppe der 18- bis 39-Jährigen im Vergleich zu den höheren Altersgruppen signifikant häufiger betroffen. Anders als körperliche Gewalterfahrungen sind psychische Gewalterfahrungen - vor allem hinsichtlich der Tä- 


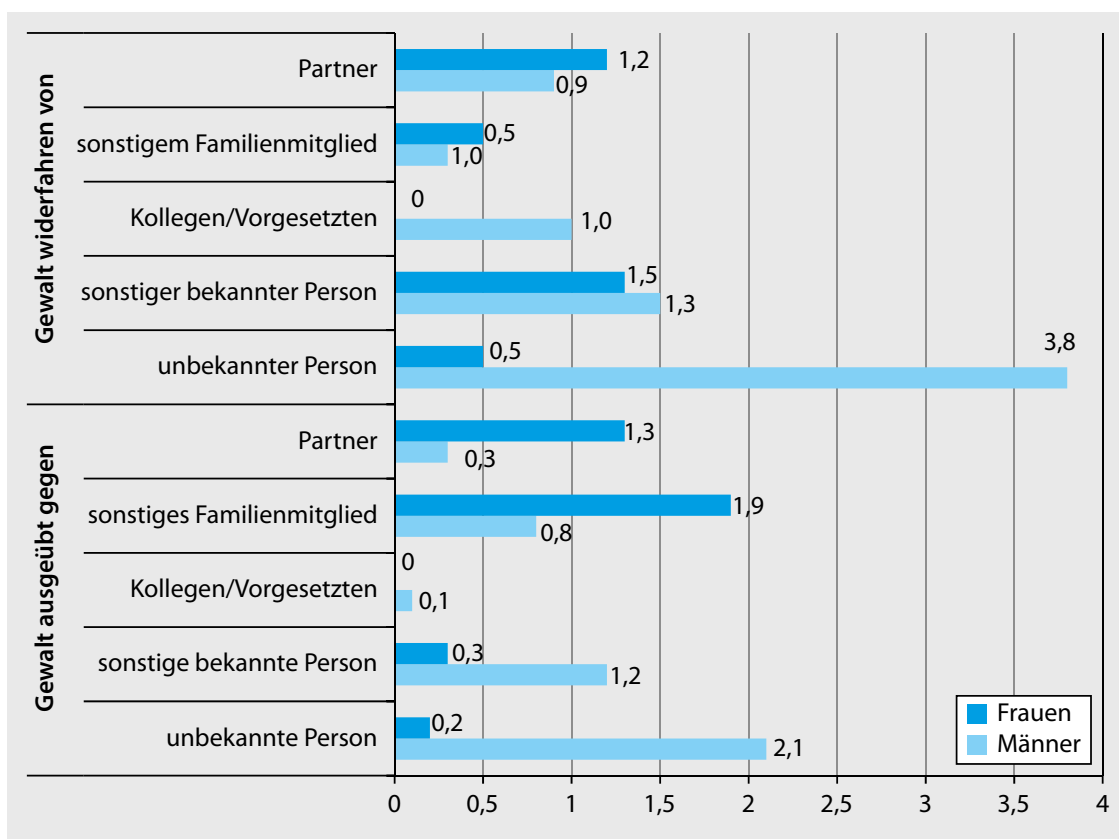

Abb. $1 \Delta$ Häufigkeiten von Erfahrungen körperlicher Gewalt nach Konfliktpartner (letzte 12 Monate, Mehrfachantworten möglich)

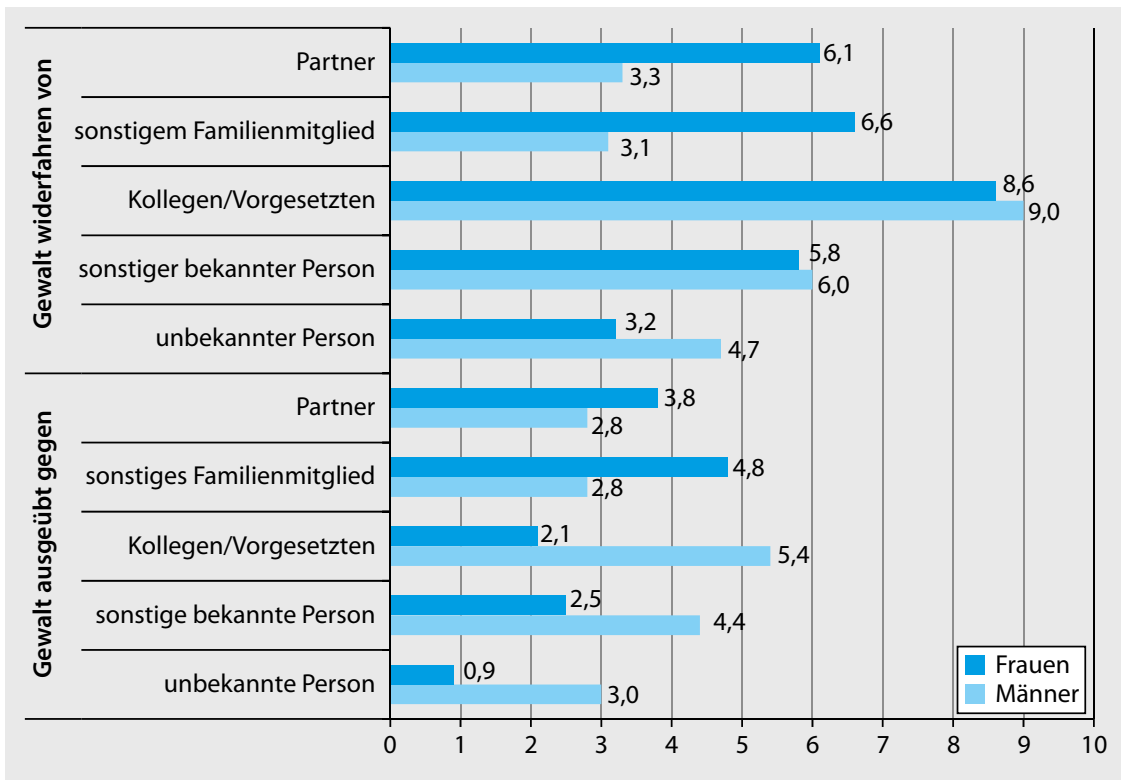

Abb. $2 \Delta$ Häufigkeiten von Erfahrungen psychischer Gewalt nach Konfliktpartner (letzte 12 Monate, Mehrfachantworten möglich)

tererfahrungen - über die verschiedenen sozialen Statusgruppen annähernd gleich verteilt. Bezüglich einer Viktimisierung durch psychische Gewalt gaben Männer mit niedrigem Sozialstatus tendenziell häufiger als Frauen an, Opfer psychischer Gewalt geworden zu sein. Bei Teilnehmenden mit einem mittleren und hohen Sozialstatus waren Frauen dagegen deutlich häufiger betroffen als Männer. Bei Personen mit mittlerem
Sozialstatus war der Geschlechtsunterschied signifikant.

\section{Körperliche Gewalt nach \\ Sozialraum/Konfliktpartner}

Laut Selbstauskunft gaben Frauen mit 1,2\% geringfügig häufiger an, Opfer körperlicher Angriffe durch ihren Partner geworden zu sein, als Männer mit 0,9\% $(\mathrm{p}=0,404)(\bullet$ Abb. 1). Ebenso berichteten sie tendenziell häufiger von körperlichen Angriffen sonstiger Familienangehöriger $(1,0$ vs. $0,5 \% ; p=0,552)$. Von Kollegen oder Vorgesetzten körperlich angegriffen worden zu sein gaben $1 \%$ der Männer, jedoch keine Frau an. Männer berichteten tendenziell häufiger als Frauen von körperlichen Angriffen sonstiger, ihnen bekannter Personen (1,5 vs. $1,3 \%$; $\mathrm{p}=0,644$ ) sowie unbekannter Personen (3,8\% vs. $0,5 \% ; \mathrm{p}<0,001)$.

Hinsichtlich der Ausübung körperlicher Partnergewalt berichteten Frauen $(1,3 \%)$ jedoch signifikant höhere Prävalenzen als Männer $(0,3 \% ; p=0,003)$ (• Abb. 1). Frauen berichteten doppelt so häufig körperliche Gewaltausübung gegen sonstige Familienmitglieder (Frauen: 1,9 vs. Männer: $0,8 \% ; p=0,001)$. Körperliche Gewalt am Arbeitsplatz übten 0,1\% der Männer, aber keine Frau aus. Die Ausübung körperlicher Gewalt gegenüber bekannten $(1,2$ vs. $0,3 \%$; $=0,004)$ oder zuvor unbekannten Personen (2,1 vs. $0,2 \%$; $\mathrm{p}<0,001)$ betraf hingegen Männer signifikant häufiger.

\section{Psychische Gewalt nach Sozialraum/Konfliktpartner}

Von ihrem Partner abgewertet, beleidigt, bedroht, beschimpft, schikaniert oder unter Druck gesetzt worden zu sein gaben mit $6,1 \%$ nahezu doppelt so viele Frauen wie Männer an $(\mathrm{p}<0,001)(\bullet$ Abb. 2). Annähernd gleiche Häufigkeiten und ein identisches Geschlechtsverhältnis zeigen sich auch bei psychischen Viktimisierungserfahrungen durch sonstige Familienangehörige $(\mathrm{p}<0,001)$. Etwa jede elfte Frau und jeder elfte Mann gaben an, psychische Gewalt am Arbeitsplatz durch Kollegen oder Vorgesetzte ( $p=0,728)$ erfahren zu haben. Diese Form psychischer Viktimisierung wurde damit am häufigsten sowohl von Männern als auch Frauen genannt. Psychische Gewalt durch sonstige bekannte Personen wurde mit ca. $6 \%$ etwa gleich häufig von Männer wie von Frauen angegeben $(\mathrm{p}=0,096)$; psychische Gewalt durch zuvor unbekannte Personen wurde dagegen signifikant häufiger von Männern berichtet $(\mathrm{p}=0,019)$.

Frauen gaben tendenziell häufiger an, Ausübende psychischer Gewalt gegenüber ihrem Partner gewesen zu sein $(\mathrm{p}=0,142)$. 
Tab. 3 Starke oder sehr starke Beeinträchtigung des Befindens durch Gewaltopfererfahrung bei Frauen und Männern in Deutschland nach Altersgruppen und Sozialstatus (DEGS1)

\begin{tabular}{|lllllll}
\hline & \multicolumn{5}{c}{ Körperliche Gewalt } & \multicolumn{3}{l}{ Psychische Gewalt } \\
\cline { 2 - 7 } & Frauen \% (95\%-KI) & Männer \% (95\%-KI) & Gesamt \% (95\%-KI) & Frauen \% (95\%-KI) & Männer \% (95\%-KI) & Gesamt \% (95\%-KI) \\
\hline Gesamt & $52,2(39,5-64,7)$ & $83,2(73,5-89,9)$ & $72,5(64,6-79,2)$ & $49,7(44,1-55,3)$ & $70,0(64,7-74,9)$ & $59,3(55,3-63,2)$ \\
\hline Altersgruppen & & & & \\
\hline 18 bis 29 Jahre & $66,5(47,6-81,3)$ & $86,6(75,9-93,0)$ & $80,5(71,3-87,2)$ & $61,8(51,8-70,8)$ & $84,8(75,9-90,8)$ & $72,8(66,7-78,1)$ \\
\hline 30 bis 44 Jahre & $44,1(22,4-68,4)$ & $76,1(44,0-92,8)$ & $63,3(43,6-79,3)$ & $72,8(62,7-80,9)$ & $45,8(35,8-56,1)$ & $59,6(52,2-66,6)$ \\
\hline 45 bis 64 Jahre & $25,5(10,2-50,7)$ & $77,9(51,3-91,4)$ & $55,6(36,4-73,2)$ & $40,6(33,1-48,7)$ & $47,8(38,4-57,3)$ & $43,7(37,9-49,6)$ \\
\hline Sozialstatus & & & & & \\
\hline Niedrig & $94,2(77,6-98,7)$ & $57,1(32,5-78,6)$ & $79,5(64,3-89,3)$ & $45,2(32,5-58,6)$ & $64,3(50,7-75,9)$ & $53,8(44,5-62,8)$ \\
\hline Mittel & $49,6(34,0-65,3)$ & $82,1(68,3-90,7)$ & $71,1(60,1-80,0)$ & $49,9(42,9-57,0)$ & $70,5(63,6-76,7)$ & $59,9(54,9-64,7)$ \\
\hline Hoch & $69,3(43,0-87,1)$ & $43,4(17,8-73,1)$ & $61,7(41,0-78,9)$ & $49,4(38,5-60,4)$ & $73,7(63,1-82,2)$ & $60,8(52,1-68,9)$ \\
\hline KI Konfidenzintervall. & & & & &
\end{tabular}

Ebenso gaben Frauen häufiger an, psychische Gewalt gegenüber einem sonstigen Familienmitglied ausgeübt zu haben ( $p=0,005)$. Gegenüber Kollegen oder Vorgesetzten $(\mathrm{p}<0,001)$ sowie gegenüber sonstigen bekannten $(\mathrm{p}=0,001)$ oder $\mathrm{zu}$ vor unbekannten Personen $(\mathrm{p}<0,001)$ übten hingegen Männer signifikant häufiger psychische Gewalt aus.

\section{Viktimisierung und Gewaltausübung in mehreren Sozialräumen}

Hinsichtlich körperlicher Gewaltopfererfahrungen gaben insgesamt 169 $(87,1 \%)$ von 194 Männern mit Gewaltopfererfahrung einen, 25 (12,9\%) 2 oder mehr Konfliktpartner an. 98 (96,1\%) von 102 Frauen berichteten von einer Opferwerdung durch nur einen und $4(3,9 \%)$ Frauen durch 2 oder mehr Konfliktpartner (Daten nicht in Tabelle). Hinsichtlich körperlicher Gewaltausübung gaben $91(78,8 \%)$ von 116 Männern mit Täterhistorie an, gegenüber nur einem Konfliktpartner gewalttätig gewesen zu sein, und $25(21,2 \%)$ gegenüber 2 oder mehreren Konfliktpartnern. Bezüglich psychischer Opfererfahrung gaben $415(76,2 \%)$ von 545 Männern eine Viktimisierung durch einen und $130(23,8 \%)$ eine Viktimisierung durch 2 oder mehr Konfliktpartner an. Von 624 Frauen gaben 469 $(75,1 \%)$ eine Viktimisierung durch einen und $155(24,9 \%)$ durch 2 oder mehr Konfliktpartner an. Insgesamt $242(68,1 \%)$ von 355 Männern und 222 (73,0\%) von 304 Frauen gaben die Ausübung psychischer Gewalt gegenüber nur einem Kon- fliktpartner an, 113 (31,9\%) Männer und 13 (30,0\%) Frauen gaben an, gegenüber 2 oder mehreren Konfliktpartnern psychisch gewalttätig geworden zu sein.

\section{Belastungserleben durch Viktimisierung nach Geschlecht, Alter und Sozialstatus}

Generell ist das Belastungserleben durch Gewaltopfererfahrungen hoch, wobei das Befinden bei der Viktimisierung durch körperliche Gewalt stärker beeinträchtigt ist als bei einer psychischen Viktimisierung. Bezüglich körperlicher Gewaltopfererfahrung gaben insgesamt fast drei Viertel, bezüglich psychischer Gewaltopfererfahrungen ca. $60 \%$ der Befragten eine starke oder sehr starke Beeinträchtigung ihres Befindens an (• Tab. 3). Männer gaben insgesamt zu einem deutlich und signifikant höheren Prozentsatz als Frauen starke oder sehr starke Beeinträchtigungen durch Viktimisierungserfahrungen an. Dies gilt sowohl für die körperlichen als auch für die psychischen Gewaltopfererfahrungen über alle Altersgruppen. Eine Ausnahme gibt es bei Frauen im mittleren Altersbereich von 30 bis 44 Jahren, die signifikant häufiger starke und sehr starke Beeinträchtigungen des Befindens nach einer psychischen Viktimisierung angaben als Männer. Bei einem niedrigen sowie hohen Sozialstatus gaben Frauen umgekehrt deutlich häufiger an, durch körperliche Gewaltopfererfahrung in ihrem Befinden beeinträchtigt zu sein als die Männer (• Tab. 3).
Belastungserleben nach Geschlecht und Konfliktpartner/ Sozialraum

Im Folgenden werden Beeinträchtigungen des Befindens bezogen auf die jeweiligen Konfliktpartner wiedergegeben (Daten nicht in Tabelle). Die Angaben beziehen sich ausschließlich auf die Personen, die als Täter bzw. Opfer jeweils nur einen Konfliktpartner angegeben hatten. Die Zahlen sind daher entsprechend vorsichtig zu interpretieren; allerdings ergab die exploratorische Analyse für Personen, die mehrere Konfliktpartner benannt hatten, in der Tendenz ähnliche Ergebnisse.

Männer, die angaben, Opfer von körperlicher Gewalt durch den Partner geworden zu sein, gaben mit $83,1 \%$ deutlich häufiger starke oder sehr starke Beeinträchtigungen ihres Befindens als Frauen mit 46,7\% ( $p=0,072)$. Bezüglich körperlicher Gewalt durch sonstige Familienangehörige gaben alle Männer (100\%) und $36,9 \%(\mathrm{p}=0,088)$ der Frauen starke oder sehr starke Beeinträchtigungen an. Körperliche Gewalt am Arbeitsplatz führte bei $80,9 \%$ der Männer zu starken oder sehr starken Beeinträchtigungen (Frauen gaben keine körperlichen Gewalterfahrungen am Arbeitsplatz an). Ein analoges Bild ergab sich für körperliche Gewaltopfererfahrungen durch bekannte (Frauen: 57,1\%, Männer: $84,1 \%$; $p=0,089)$ und unbekannte Personen (Frauen: 69,9\%, Männer: $86,6 \% ; \mathrm{p}=0,313)$.

Das Belastungserleben hinsichtlich psychischer Gewalt durch den Partner ist bei beiden Geschlechtern insgesamt 
Tab. 4 Unrechtserleben nach Ausübung von Gewalt bei Männern und Frauen in Deutschland, stratifiziert nach Altersgruppen und Sozialstatus (DEGS1)

\begin{tabular}{|lllllll}
\hline & \multicolumn{5}{c}{ Körperliche Gewalt } & \multicolumn{3}{l}{ Psychische Gewalt } \\
\cline { 2 - 7 } & Frauen \% (95\%-KI) & Männer \% (95\%-KI) & Gesamt \% (95\%-KI) & Frauen \% (95\%-KI) & Männer \% (95\%-KI) & Gesamt \% (95\%-KI) \\
\hline Gesamt & $88,9(78,4-94,6)$ & $47,8(36,3-59,4)$ & $67,0(58,5-74,5)$ & $76,7(69,6-82,5)$ & $60,6(53,4-67,3)$ & $68,1(63,2-72,7)$ \\
\hline Altersgruppen & & & & & \\
\hline 18 bis 29 Jahre & $80,3(58,3-92,2)$ & $41,5(27,3-57,3)$ & $55,2(42,2-67,5)$ & $75,2(63,2-84,3)$ & $56,2(44,4-67,4)$ & $64,8(55,8-73,0)$ \\
\hline 30 bis 44 Jahre & $96,5(87,7-99,1)$ & $63,8(35,1-85,1)$ & $86,0(71,8-93,7)$ & $72,2(57,4-83,4)$ & $66,7(53,1-77,9)$ & $69,2(59,6-77,4)$ \\
\hline 45 bis 64 Jahre & $89,0(62,0-97,6)$ & $56,7(24,9-83,8)$ & $70,6(44,3-87,8)$ & $83,8(73,7-90,6)$ & $59,5(45,5-72,1)$ & $72,1(62,5-80,0)$ \\
\hline Sozialstatus & & & & & \\
\hline Niedrig & $84,7(39,9-97,9)$ & $55,5(33,3-75,6)$ & $64,9(46,5-79,8)$ & $69,5(47,2-85,4)$ & $55,4(37,2-72,3)$ & $34,8(23,2-48,4)$ \\
\hline Mittel & $91,2(77,6-96,8)$ & $48,9(34,4-63,7)$ & $70,8(58,9-79,8)$ & $76,7(67,9-83,6)$ & $58,7(49,1-67,7)$ & $67,0(60,4-73,0)$ \\
\hline Hoch & $81,7(56,4-93,9)$ & $28,4(11,9-53,8)$ & $52,9(32,3-72,6)$ & $81,3(66,5-90,5)$ & $70,6(54,5-82,9)$ & $75,1(63,8-83,8)$ \\
\hline KI Konfidenzintervall. & & & & &
\end{tabular}

geringer. Jedoch berichteten beide Geschlechter annähernd gleich häufig Beeinträchtigungen des Befindens (Frauen: 49,8\%; Männer: 44,6\%; p=0,687). Hinsichtlich Gewaltopfererfahrungen durch sonstige Familienmitglieder (Frauen: 38,8\%, Männer: 65,5\%; $\mathrm{p}=0,103$ ), durch Kollegen oder Vorgesetzte am Arbeitsplatz (Frauen: 48,9\%, Männer: 62,1\%; $\mathrm{p}=0,050$ ), durch bekannte (Frauen: 60,5\%, Männer: 87,7\%; $p=0,001)$ oder unbekannte Personen (Frauen: 71,7\%, Männer: 86,2\%; $p=0,169)$ waren Männer häufiger in ihrem Befinden beeinträchtigt.

\section{Unrechtserleben bei Gewaltausübung nach Geschlecht, Alter und Sozialstatus}

Unrechtserleben im Sinne eines schlechten Gewissens nach Ausübung von Gewalt gaben sowohl für körperliche als auch für psychische Gewalthandlungen etwa zwei Drittel aller Befragten an (- Tab. 4). Jedoch gibt es hier deutliche Geschlechtsunterschiede: Frauen gaben mit insgesamt $88,9 \%$ bei körperlicher Gewaltausübung und $76,7 \%$ bei psychischer Gewaltausübung deutlich und signifikant häufiger ein schlechtes Gewissen an als Männer (47,8\% respektive 60,6\%). Diese Unterschiede zeigen sich durchgängig über die Altersgruppen und den Sozialstatus, erreichen jedoch für psychische Gewaltausübung überwiegend nicht die statistische Signifikanz.
Unrechtserleben nach Geschlecht und Konfliktpartner/ Sozialraum

Eine weitere Differenzierung ergibt sich aus der Betrachtung nach dem jeweiligen Konfliktpartner (Daten nicht in Tabelle). Dargestellt sind wiederum nur Angaben der Personen, die als Täter bzw. Opfer jeweils nur einen Konfliktpartner angegeben hatten. Auch hier ergab die entsprechende Sensitivitätsanalyse für Personen, die mehrere Konfliktpartner benannt hatten, keine substanziellen Abweichungen. Dennoch sind diese Zahlen entsprechend vorsichtig zu interpretieren.

Nach körperlicher Gewaltausübung gegenüber dem Partner ergeben sich für beide Geschlechter annähernd gleich hohe Raten für ein Unrechtserleben (Frauen: $74,6 \%$; Männer 76,5\%, p=0,921). Unterschiede gab es jedoch hinsichtlich körperlicher Angriffe auf sonstige Familienangehörige (Frauen: 99,0\%; Männer 63,7\%, $\mathrm{p}<0,001)$ sowie körperlicher Gewaltausübung gegenüber bekannten (Frauen: $82,2 \%$; Männer: $30,1 \% ; \mathrm{p}=0,078)$ und unbekannten Personen (Frauen: 64,4\%; Männer: 35,2\%; $\mathrm{p}=0,037$ ).

Die Häufigkeit von schlechtem Gewissen nach psychischer Gewaltausübung gegenüber dem Partner (Frauen 89,4\%, Männer: $86,4 \%$; $p=0,779)$, sonstigen $\mathrm{Fa}$ milienangehörigen (Frauen $83,4 \%$, Männer: 71,5\%; $p=0,302)$, Kollegen/Vorgesetzten am Arbeitsplatz (Frauen 58,4\%, Männer: 52,4\%; $p=0,650$ ) war bei Frauen und Männern annähernd gleich verteilt. Schlechtes Gewissen nach psychischer Gewaltausübung gegenüber bekannten
(Frauen 64,4\%, Männer: 35,2\%; p=0,039) oder unbekannten Personen (Frauen $60,8 \%$, Männer: $37,0 \% ; p=0,323$ ) war bei Frauen jedoch deutlich häufiger.

\section{Diskussion}

In der Studie zur Gesundheit Erwachsener in Deutschland (DEGS1) wurde die 12-Monats-Prävalenz individueller Erfahrungen von körperlicher und psychischer Gewalt bei insgesamt 5939 Frauen und Männern im Alter von 18 bis 64 Jahren erfasst. Etwa jeder 20. Erwachsene war in den letzten 12 Monaten als Opfer von körperlicher Gewalt betroffen, die 12-Monats-Prävalenz für Tätererfahrungen von körperlicher Gewalt lag insgesamt bei 3,4\%. Vergleichbare 12-Monats-Raten von $2-4 \%$ wurden auch in anderen internationalen Studien gefunden $[26,27]$. Etwa 20\% der Teilnehmenden berichtete über Opfererfahrungen durch psychische Gewalt, jeder zehnte Teilnehmende berichtete, psychische Gewalt ausgeübt zu haben. Männer waren insgesamt häufiger sowohl unter den Opfern als auch unter den Tätern körperlicher Gewalt, während sich die Raten für psychische Gewalt hinsichtlich des Geschlechts jeweils nicht unterschieden. Unserer Kenntnis zufolge gibt es bislang keine Studie, die Daten zu psychischer Gewalt auf repräsentativer, bevölkerungsbezogener Basis für Frauen und Männer als Täter- und Opfererfahrung erhoben hat. Coker et al. [28] fanden in einem Survey, der ausschließlich Frauen untersuchte, dass psychische Gewaltopfererfahrungen ebenso stark mit nega- 
tiven Konsequenzen für die Gesundheit assoziiert waren wie körperliche. Körperliche Gewalt ist vor allem ein Phänomen des frühen Erwachsenenalters: die Häufigkeiten sowohl von Opfer- als auch Tätererfahrungen gehen bei beiden Geschlechtern mit zunehmendem Alter stark zurück, was entwicklungspsychologisch plausibel ist. Das frühe Erwachsenenalter ist gekennzeichnet durch Transitionsprozesse wie die Ablösung vom Elternhaus, berufliche Ausbildung und Entwicklung, Familiengründung und Übernahme gesellschaftlicher Verantwortung [29], die mit Unsicherheiten und damit mit höheren Konfliktrisiken einhergehen. Dass körperliche Gewalt dem sozialen Status gegenläufig assoziiert ist, entspricht ebenfalls der Studienlage [30, 31].

Opfer von körperlicher Gewalt durch den Partner in den letzten 12 Monaten wurden 1,2\% der Frauen und 0,9\% der Männer. Vergleichbare Häufigkeiten (Frauen: 1,1\%; Männer: 0,6\%) wurden von im US-amerikanischen „National Violence Against Women Survey“ [32] sowie in einer bevölkerungsbezogenen Repräsentativstudie in Irland (Frauen: 1,4\%: Männer: 1,4\%) gefunden [33]. Den Ergebnissen unserer Studie zufolge waren Frauen auch tendenziell häufiger Opfer von körperlicher und psychischer Gewalt durch sonstige Familienangehörige. Dies ist bislang wenig erforscht. Eine aktuelle Analyse sämtlicher staatlich registrierter Vorfälle Kind-initiierter Gewaltausübung gegenüber den Eltern in den USA im Zeitraum von 1995-2005 zeigte, dass nichttödliche Angriffe mehrheitlich von männlichen Personen $(62,6 \%)$ begangen wurden, die Opfer jedoch mehrheitlich weiblich $(71,9 \%)$ waren [34].

Bemerkenswert ist, dass in dieser Studie Frauen häufiger als Männer Ausübende körperlicher Partnergewalt, aber auch Ausübende von Gewalt gegenüber sonstigen Familienmitgliedern waren. Weibliche Gewalt scheint nach den Daten von DEGS1 somit schwerpunktmäßig im häuslichen Bereich lokalisiert zu sein. Hinsichtlich der in bevölkerungsbezogenen Studien häufig gefundenen gleichen, zum Teil höheren Raten von physischer Partnergewalt von Frauen gegenüber Männern ist häufig argumentiert worden, dass Partnergewalt von Frauen pri- mär in Selbstverteidigungsabsicht gegenüber einem männlichen Partner-Aggressor ausgeübt wird [35]. Carney und Kollegen [36] stellen diesen Erklärungsansatz in einem aktuellen Literaturreview jedoch infrage. Aus den von ihnen zusammengetragenen Ergebnissen repräsentativer, bevölkerungsbezogener, klinischer und entwicklungspsychologischer Studien schließen sie, dass weibliche wie männliche Partnergewalt nicht nur annähernd gleich häufig vorkommt, sondern sowohl von Männern als auch von Frauen annähernd gleich häufig initiiert wird, und dass sich Täterinnen und Täter hinsichtlich ihrer psychosozialen Charakteristika stärker ähneln, als bislang angenommen [36].

Die DEGS1-Daten zeigen, dass vor allem körperliche, aber auch psychische Gewaltopfererfahrungen als sehr belastend erlebt werden. Dabei ist das Belastungserleben bei Männern jeweils deutlich höher als bei Frauen. Dies kann als Hinweis auf eine fehlende sozial akzeptierte Opferrolle für Männer interpretiert werden [37]. Bemerkenswert ist, dass Männer, die als Opfer von familiärer und Partnergewalt betroffen waren, ein besonders starkes Belastungserleben angaben. Unrechtserleben in Sinne eines schlechten Gewissens nach der Ausübung von körperlicher oder psychischer Gewalt wurde insgesamt häufiger von Frauen als von Männern angegeben, allerdings gaben bezüglich ausgeübter Partnergewalt Männer und Frauen gleich häufig ein schlechtes Gewissen an.

Nach den Daten von DEGS1 wurden ausschließlich Männer (1\%) Opfer von körperlicher und etwa $9 \%$ beider Geschlechter Opfer von psychischer Gewalt am Arbeitsplatz. Gewalt am Arbeitsplatz stellt nicht nur ein unmittelbares Gesundheitsrisiko dar, sondern hat darüber hinaus negative Auswirkungen auf die Leistungsfähigkeit und Gesundheit Betroffener und verursacht auf diese Weise wirtschaftliche Verluste $[38,39,40]$. Nach den Ergebnissen des 2007 in 31 europäischen Ländern durchgeführten „Fourth European Survey on Working Conditions" (ESWC) waren 5\% der Arbeitnehmer in den letzten 12 Monaten Opfer körperlicher Gewalt durch Personen der Belegschaft [41]. Für Deutschland lagen die Prävalenzen für körperliche Gewalt in diesem Survey bei ca. 6\% und damit höher als in DEGS1. Ein Grund hierfür könnten unter anderem Stichprobeneffekte sein (im ESWC wurden ausschließlich Arbeitnehmer befragt, die Daten von DEGS1 beziehen sich auf die Gesamtbevölkerung). Da Frauen insgesamt weniger umfangreich erwerbstätig sind als Männer [42], implizieren die DEGS1-Ergebnisse, dass Frauen - in Relation zum Umfang der Erwerbstätigkeit - häufiger Opfer von psychischer Gewalt am Arbeitsplatz sind als Männer. Diese Interpretation wird durch die Ergebnisse des bundesdeutschen Mobbing-Reports 2002 gestützt [43].

Laut polizeilicher Kriminalstatistik wird in Deutschland Gewalt im öffentlichen Raum in Form von Raub, räuberischer Erpressung oder räuberischer Angriffe auf Kraftfahrer zu einem Großteil von unbekannten, versuchte oder vollendete Körperverletzung jedoch vorwiegend von dem Opfer bekannten Tätern begangen [11]. Täter sowie Opfer dieser angezeigten Gewalthandlungen sind vorwiegend Männer. Diese Tendenz drückt sich für Opfer- und Tätererfahrung ebenfalls in den DEGS1-Daten aus. In internationalen Studien wird Gewalt im außerhäuslichen Raum („community violence“) nicht immer klar gegen Gewaltereignisse im häuslichen Bereich und am Arbeitsplatz abgegrenzt, da neben Gewaltereignissen im Wohnumfeld oft auch familiäre Gewalt definitorisch eingeschlossen wird (z. B. $[5,44])$. Personen, die infolge eines gewalttätigen Angriffs im öffentlichen Raum verletzt werden, weisen allerdings neben den körperlichen Verletzungsfolgen auch psychische Beeinträchtigungen auf. Ramchand und Kollegen [45] konnten zeigen, dass der Heilungsprozess nach körperlicher Traumatisierung durch Gewalt umso ungünstiger verläuft, je höher die psychische Belastung unmittelbar nach einem gewalttätigen Angriff ist.

\section{Einschränkungen und Stärken}

Diese Studie erhebt nicht den Anspruch, ein vollständiges Abbild der Gewaltereignisse in der deutschen Erwachsenenbevölkerung vorzulegen. Insbesondere 
ist hierbei auf die fehlende Erhebung sexueller aber auch sozial-relationaler Gewalt zu verweisen, auf die, wie eingangs ausgeführt, aus Durchführbarkeits- sowie aus befragungsökonomischen Gründen verzichtet werden musste. Aufgrund der Datenlage ist anzunehmen, dass sexuelle Gewalt in höherem Maße Frauen betrifft $[8,9]$, während sozial-relationale Gewalt in bedeutsamer Weise auch Männer betreffen dürfte [37]. Aus dem DEGS1-Gewaltscreening ergeben sich weiterhin keine Informationen zur Dynamik des Konfliktgeschehens oder zu möglichen Verletzungsfolgen von Gewalthandlungen. Zudem war der Gewaltscreener zum Zeitpunkt seines Einsatzes noch nicht klinisch validiert. Die Erhebung von sensitiven Themen wie Gewalterfahrungen birgt stets die Gefahr von Antworttendenzen wie der sozialen Erwünschtheit oder eines Underreportings. Die aufgezeigte gute Übereinstimmung der DEGS1-Ergebnisse mit denen vergleichbarer internationaler Studien legt jedoch nahe, dass dies in DEGS1 keine größere Rolle spielt als in anderen Studien auch. Eine weitere Einschränkung stellt die Begrenzung der Erhebung auf den Altersbereich von 18 bis 64 Jahren dar, sodass die Public-Health-relevante Thematik von Gewalterfahrungen im höheren Lebensalter nicht untersucht werden konnte. Dennoch stellen die Daten einen wichtigen Zugewinn zur aktuellen Forschungslage zur Gewalt in Deutschland dar. So wurden erstmalig körperliche und psychische Gewaltereignisse bei Männern und Frauen als Täter- und Opfererfahrung bundesweit repräsentativ erhoben. Durch die Implementierung des Gewaltscreenings in den bundesweiten Gesundheitssurvey DEGS1 wurde der seit Langem erhobenen Forderung, Gewalt in der Public-Health-Forschung stärker zu berücksichtigen [46], entsprochen. Die DEGS1-Stichprobe bietet die Möglichkeit, zukünftig eine Vielzahl weiterer körperlicher und psychischer Gesundheitsparameter mit Gewalterfahrungen in Beziehung zu setzen. Ein Vorteil ist auch, dass mit dem Gewaltscreening nicht nur häusliche bzw. Partnergewalt erfasst wurde, sondern das Spektrum der Sozialräume auf Gewalterfahrungen am Arbeitsplatz sowie auf den außerhäuslichen Be- reich erweitert wurde. Dies ermöglicht einen umfassenderen Blick auf das Phänomen Gewalt und seine gesundheitlichen Korrelate.

\section{Fazit und Schlussfolgerungen}

Gewalterfahrungen sind ein oft einschneidendes, mitunter schicksalbestimmendes Gesundheits- und Lebensrisiko für Männer und Frauen. Während es, nicht zuletzt angestoßen durch die Ergebnisse der feministischen Gewaltforschung, bereits eine hohe Aufmerksamkeit und vergleichsweise gut entwickelte Hilfestrukturen für gewaltbetroffene Frauen gibt, sind, trotz der Tatsache, dass Männer insgesamt häufiger Opfer von körperlicher Gewalt werden als Frauen, Gewaltopfererfahrungen von Männern im gesellschaftlichen und wissenschaftlichen Diskurs wenig repräsentiert. Es wäre daher wünschenswert, wenn sich im öffentlichen Bewusstsein eine höhere Sensibilität und Empathie für Männer, die Opfer von Gewalthandlungen wurden, entwickelte und Opfererfahrung von Männern nicht länger als „unmännlich“ stigmatisiert würden [47]. Die soziale Akzeptanz einer männlichen Opferrolle eröffnete die Möglichkeit einer stärkeren Verankerung von Opfererfahrungen in der männlichen Selbstwahrnehmung sowie in männlichen Selbstkonzepten. Die Themen „Frauen als Gewalttäterinnen“ und „Männer als Gewaltopfer" sind gesellschaftlich noch weitgehend tabuisiert und werden erst allmählich von der Gewalt- und Genderforschung aufgegriffen. In der Vergangenheit wurde eine intensive Kontroverse um die Frage geführt, ob beide Geschlechter gleich häufig oder ob Frauen häufiger und schwerer als Opfer von Gewalthandlungen betroffen sind und ob Frauen auch oder sogar annähernd gleich häufig aggressiv und gewalttätig sein können wie Männer [14, 35, 48, 49]. Die Debatte erscheint aus gesundheitswissenschaftlicher Sicht wenig zielführend. In jüngerer Zeit hat sich jedoch z. B. die feministische Gewaltforschung der Möglichkeit männlicher Opfererfahrung und weiblicher Täterschaft geöffnet [50], was die
Perspektive auf einen konstruktiven Diskurs im Sinne der von Gewalt Betroffenen eröffnet. Tatsächlich sollte jegliche Form von Gewaltausübung sozial klar zurückgewiesen und den Gewaltopfern - weiblichen und männlichen - effektive Unterstützung zuteil werden. Dabei sollte jedoch nicht übersehen werden, dass auch Täterinnen und Täter angemessener psychosozialer Hilfe bedürfen.

\section{Korrespondenzadresse}

\section{R. Schlack}

Abteilung für Epidemiologie und

Gesundheitsmonitoring,

Robert Koch-Institut

General-Pape-Str. 62-66, 12101 Berlin

SchlackR@rki.de

Danksagung. Die Autoren danken Frau Dr. Monika Schröttle, Universität Bielefeld und Universität Gießen, für ihre Beratung bei der Instrumentenentwicklung für das Gewaltscreening.

Finanzierung der Studie. Die Studie wurde mit Mitteln des Robert Koch-Instituts und des Bundesministeriums für Gesundheit finanziert.

Interessenkonflikt. Der korrespondierende Autor gibt für sich und seine Koautoren an, dass kein Interessenkonflikt besteht.

\section{Literatur}

1. Imbusch P (2002) Der Gewaltbegriff. In: Heitmeyer W, Hagan J (Hrsg) Internationales Handbuch der Gewaltforschung. Westdeutscher, Wiesbaden, S 26-57

2. Selg H, Mees U, Berg D (1997) Psychologie der Aggressivität. 2. überarb. Aufl. Hogrefe, Göttingen

3. Fang X, Corso PS (2007) Child maltreatment, youth violence, and intimate partner violence: developmental relationships. Am J Prev Med 33:281-290

4. Manly JT, Kim JE, Rogosch FA, Cicchetti D (2001) Dimensions of child maltreatment and children's adjustment: contributions of developmental timing and subtype. Dev Psychopathol 13:759782

5. Scarpa A (2001) Community violence exposure in a young adult sample: lifetime prevalence and socioemotional effects. J Interpers Violence 16:36-53

6. Sundaram V, Helweg-Larsen K, Laursen B, Bjerregaard $P$ (2004) Physical violence, self rated health, and morbidity: is gender significant for victimisation? J Epidemiol Community Health 58:65-70

7. Sirotich F (2008) Correlates of crime and violence among persons with mental disorder: an evidence-based review. Brief Treat Crisis Interv 8:171-194

8. Coker AL, Davis KE, Arias I et al (2002) Physical and mental health effects of intimate partner violence for men and women. Am J Prev Med 23:260-268 
9. Robert Koch-Institut (Hrsg) (2008) Gesundheitliche Folgen von Gewalt unter besonderer Berücksichtigung von häuslicher Gewalt gegen Frauen. Gesundheitsberichterstattung des Bundes. Heft 42. RKI, Berlin

10. Dobash RE, Dobash RP (1977-1978) Wives: the „appropriate" victims of marital violence. Victimology 2:426-442

11. Bundeskriminalamt (Hrsg) (2010) Polizeiliche Kriminalstatistik 2011. Wiesbaden

12. Archer J (2000) Sex differences in aggression between heterosexual partners: a meta-analytic review. Psychol Bull 126:651-680

13. Fiebert MS (2004) References examing assaults by women on their spouses or male partners: an annotated bibliography. Sex Cult 8:140-177

14. Straus MA (2004) Women's violence toward men is a serious social problem. In: Gelles RJ, Loseke DR (Hrsg) Current controversies on family violence, 2. Aufl. Sage Publications, Newbury Park S 55-77

15. Kurth BM, Lange C, Kamtsiuris $P$, Hölling H (2009) Gesundheitsmonitoring am Robert Koch-Institut. Sachstand und Perspektiven. Bundesgesundheitsbl Gesundheitsforsch Gesundheitsschutz 52:557-570

16. Gößwald A, Lange M, Kamtsiuris $P$, Kurth BM (2012) DEGS: Studie zur Gesundheit Erwachsener in Deutschland. Bundesweite Quer- und Längsschnittstudie im Rahmen des Gesundheitsmonitorings des Robert Koch-Instituts. Bundesgesundheitsbl Gesundheitsforsch Gesundheitsschutz 55:775-780

17. Kurth BM (2012) Das RKI-Gesundheitsmonitoring - was es enthält und wie es genutzt werden kann. Public Health Forum 20(76):4.e1-4.e3

18. Scheidt-Nave C, Kamtsiuris P, Gößwald A et al (2012) German Health Interview and Examination Survey for Adults (DEGS) - design, objectives and implementation of the first data collection wave. BMC Public Health 12:730

19. Kamtsiuris $P$, Lange $M$, Hoffmann R et al (2013) Die erste Welle der Studie zur Gesundheit Erwachsener in Deutschland (DEGS1). Stichprobendesign, Response, Gewichtung und Repräsentativität. Bundesgesundheitsbl Gesundheitsforsch Gesundheitsschutz 56:620-630

20. Robert Koch-Institut (Hrsg) (2009) DEGS: Studie zur Gesundheit Erwachsener in Deutschland - Projektbeschreibung. Beiträge zur Gesundheitsberichterstattung des Bundes. RKI, Berlin

21. Gößwald A, Lange M, Dölle R, Hölling H (2013) Die erste Welle der Studie zur Gesundheit Erwachsener in Deutschland (DEGS1). Gewinnung von Studienteilnehmenden, Durchführung der Feldarbeit und Qualitätsmanagement. Bundesgesundheitsbl Gesundheitsforsch Gesundheitsschutz 56:611-619

22. Lampert T, Kroll L, Müters S, Stolzenberg H (2013) Messung des sozioökonomischen Status in der Studie zur Gesundheit Erwachsener in Deutschland (DEGS1). Bundesgesundheitsbl Gesundheitsforsch Gesundheitsschutz 56:631-636

23. World Health Organization (WHO) (2001) Putting women first. Ethical and safety recommendations for research on domestic violence against women. Geneva, Switzerland

24. Gößwald $A$, Lange $M$, Kamtsiuris $P$, Kurth $B M$ (2012) DEGS: Studie zur Gesundheit Erwachsener in Deutschland. Bundesweite Quer- und Längsschnittstudie im Rahmen des Gesundheitsmonitorings des Robert Koch-Instituts. Bundesgesundheitsbl Gesundheitsforschung Gesundheitsschutz 55:775-780
25. BMFSFJ (2004) Lebenssituation, Sicherheit und Gesundheit von Frauen in Deutschland. Eine repräsentative Untersuchung zu Gewalt gegen Frauen in Deutschland. http://www.bmfsfj.de/RedaktionBMFSFJ/Abteilung4/Pdf-Anlagen/langfassung-studie-frauen-teil-eins, property=pdf,bereich $=$ bmfsfj,sprache $=$ de, $r w b=$ true.pdf $($ Zugegrif fen: 12.09.2012)

26. Gelles RJ (2002) Gewalt in der Familie. In: Heitmeyer W, Hagan J (Hrsg) Internationales Handbuch der Gewaltforschung. Westdeutscher, Wiesbaden, S 1043-1077

27. Fanslow J, Robinson E, Crengle S, Perese L (2010) Juxtaposing beliefs and reality: prevalence rates of intimate partner violence and attitudes to violence and gender roles reported by New Zealand women. Violence Against Women 16:812-831

28. Coker AL, Smith PH, Bethea L et al (2000) Physical health consequences of physical and psychological intimate partner violence. Arch Fam Med 9:451-457

29. Krampen G, Reichle B (2002) Frühes Erwachsenen alter. In: Oerter R, Montada T (Hrsg) Entwicklungspsychologie. Beltz, Weinheim, S 319-349

30. Cunradi CB, Caetano R, Schafer J (2002) Socioeconomic predictors of intimate partner violence among white, black, and hispanic couples in the United States. J Fam Violence 17:377-389

31. Weede $E$ (1981) Income inequality, average income, and domestic violence. J Conflict Resolut 25:639-654

32. Tjaden P, Thoennes N (2000) Prevalence and consequences of male-to-female and female-to-male intimate partner violence as measured by the national violence against women survey. Violence Against Women 6:142-161

33. Watson D, Parsons S (2005) Domestic abuse of women and men in Ireland. Report on the National Study of Domestic Abuse National Crime Council, Dublin

34. Walsh JA, Krienert JL (2009) A decade of child-initiated family violence. Comparative analysis of child-parent violence and parricide examining offender, victim, and event characteristics in a national sample of reported incidents, 1995-2005, J Interpers Violence 24:1450-1477

35. Kimmel MS (2002) "Gender Symmetry“ in domestic violence a substantive and methodological research review. Violence Against Women 8:13321363

36. Carney M, Butell F, Dutton D (2007). Women who perpetrate intimate partner violence: a review of the literature with recommendations for treatment. Aggress Violent Behav 12:108-115

37. BMFSFJ (2004) Gewalt gegen Männer in Deutschland. Personale Gewalterfahrnisse von Männern in Deutschland. http://www.bmfsfj.de/RedaktionBMFSFJ/Abteilung4/Pdf-Anlagen/studie-gewalt-maenner-langfassung, property $=$ pdf, bereich $=$ bmfsfi, sprache $=$ de, rwb=true.pdf (Zugegriffen: 05.08.2012)

38. Hoel H, Sparks K, Cooper CL (2001) The cost of violence/stress at work and the benefits of a violence/stress-free working environment. University of Manchester Institute of Science and Technology. Report commissioned by the Interntational Labour Organisation (ILO), Geneva. http://www.lo.org/ wcmsp5/groups/public/---ed_protect/---protrav/--safework/documents/publication/wcms_118190. pdf (Zugegriffen: 12.08.2012)
39. Di Martino V (2002) Violence at the workplace; the global response. African Newsl Occup Health Saf 12:4-6

40. Mayhew C, Chapell D (2007) Workplace violence: an overview of patterns of risk and the emotional/ stress consequences of targets. Int J Law Psychiatry 327-339

41. Parent-Thirion A, Fernández Macías E, Hurley J, Vermeylen G (2007) Fourth European Working Conditions Survey. European Foundation for the Improvement of Living and Working Conditions, Dublin. http://www.eurofound.europa.eu/pubdocs/2006/98/en/2/ef0698en.pdf (Zugegriffen: 25.03.2013)

42. Statistisches Bundesamt (2010) Mikrozensus 2009. Bevölkerung und Erwerbstätigkeit. Stand und Entwicklung der Erwerbstätigkeit in Deutschland. Wiesbaden

43. Meschkutat B, Stackelberg M, Langenoff G (2002) Der Mobbing-Report. Eine Repräsentativstudie für die Bundesrepublik Deutschland. Schriftenreihe der Bundesanstalt für Arbeitsschutz und Arbeitsmedizin. Dortmund, Berlin

44. Scarpa A, Fikretoglu D, Bowser F et al (2002) Community violence exposure in university students. J Interpers Violence 17:253-272

45. Ramchand R, Marshall GN, Schell TL et al (2008) Posttraumatic distress and physical functioning: a longitudinal study of injured survivors of community violence. J Consult Clin Psychol 76:668

46. Hagemann-White C (2001) European research on the prevalence of violence against women. Violence Against Women 7:732-759

47. Heinrich-Böll-Stiftung (Hrsg) (2002) Mann oder Opfer? Dokumentation einer Fachtagung des Forums Männer in Theorie und Praxis der Geschlechterverhältnisse und der Heinrich Böll Stiftung am 12./13. Oktober 2001 in Berlin. Heinrich-Böll-Stiftung, Berlin

48. Straus MA (2009) Why the overwhelming evidence on partner physical violence by women has not been perceived and is often denied. J Aggress Maltreat Trauma 18:552-571

49. Dobash RP, Dobash RE, Wilson M, Daly M (1992) The myth of sexual symmetry in marital violence. Soc Probl 39:71-91

50. Kavemann B (2009) Täterinnen - die Gewaltausübung von Frauen im privaten Raum im Kontext der feministischen Diskussion über Gewalt im Geschlechterverhältnis. Neue Kriminalpolitik 2:46-50 